Edición Extraordinaria. p.p. $182-193$

Memorias del VIII Encuentro Nacional de Experiencias en Enseñanza de la Biología y la Educación Ambiental. III Congreso Nacional de Investigación en Enseñanza de la Biología.

\title{
EL CINE DE CIENCIA FICCIÓN PARA LA ENSEÑANZA DE LAS CIENCIAS: ANÁLISIS DE CONTENIDO
}

\section{FOR SCIENCE EDUCATIONT THE FICTION SCIENCE FILMS: TOPICS REVIEW}

\section{Ceballos Abello, $G$.}

Licenciado en Biología De La Universidad Distrital Francisco José De Caldas, Estudiante de Maestría en Educación, Énfasis en Educación en Ciencias de la Naturaleza y la Tecnología. Grupo de Investigación BER (Biología, Enseñanza y Realidades)

Resumen: Este documento corresponde a una fase del proyecto de grado: se presenta un análisis de contenido respecto a las posibilidades que ofrece la ciencia ficción en la enseñanza y la manera como diferentes investigadores a partir de un mismo recurso como la ciencia ficción, han aprovechado su potencial con distintos fines. Para la realización del presente trabajo se seleccionaron las bases de datos Dialnet, Scielo y Eric para consultar la información (artículos) que suministraron la materia prima para la realización del análisis de contenido, la ventana de observación que se estableció corresponde a un periodo de tiempo no mayor a 10 años, las bases de datos aportaron cerca de 20000 artículos los cuales fueron categorizados; logrando reducir el número de documentos, el descriptor seleccionado para la base de datos Dialnet corresponde a "El cine de ciencia ficción para la enseñanza de las ciencias", mientras que para la base de datos Eric se seleccionó como descriptor "Enseñanza de las ciencias y ciencia ficción", en cuanto a la base de datos Scielo solo se encontró un (1) articulo correspondiente al descriptor "Ciencia ficción y enseñanza". Al final el análisis de contenido se basó en cinco (5) documentos que guardan una profunda relación con la naturaleza de la propuesta de proyecto de grado, permitiendo deducir que la CF es un instrumento versátil que ofrece varias posibilidades en el ámbito educativo.

Abstract: The document corresponds to a phase of degree; a content analysis regarding the possibilities offered by science fiction in teaching and how different researchers from the same resource as it is science fiction is presented, they have used their potential for different purposes. To carry out this work Dialnet bases, Scielo and Eric data were selected to review information (items) that provided the raw material for conducting content analysis, the observation window that corresponds to a set period of time no more than 10 years, the databases provided about 20,000 items which were categorized with which it was possible to reduce the number of documents the descriptor selected to the database Dialnet corresponds to "The science fiction films for teaching science ", while for the database descriptor Eric was selected as" science education and science fiction "about the database Scielo only 1 item found 
Edición Extraordinaria. p.p. $182-193$

Memorias del VIII Encuentro Nacional de Experiencias en Enseñanza de la Biología y la Educación Ambiental. III Congreso Nacional de Investigación en Enseñanza de la Biología.

corresponding to the descriptor" Science fiction and teaching. "At the end content analysis it was based on five documents that have a deep relationship with the nature of the project proposal degree, allowing to deduce that the $C F$ is a versatile instrument that offers several possibilities in education.

Palabras clave: Alfabetización, ciencia ficción, aprendizaje, enseñanza, recurso, motivación, metodología, ventajas, didáctica, análisis, investigación.

Keywords: Literacy, fiction science, learning, teaching, resources, motivation, methodology, advantages, teaching, analysis, research.

El presente texto o disertación corresponde a una fase del proyecto de investigación titulado "LA INVESTIGACIÓN ESCOLAR SOPORTADA EN LA CIENCIA FICCIÓN COMO ELEMENTO PARA LA ENSEÑANZA APRENDIZAJE DE LA GENÉTICA EN ESTUDIANTES DE GRADO OCTAVO DEL IED CARLOS ALBÁN HOLGUÍN", para este congreso se presenta un análisis de contenido correspondiente a las posibilidades que ofrece la ciencia ficción en la enseñanza y la manera como diferentes investigadores a partir de un mismo recurso han aprovechado su potencial con distintos fines. Para la consecución de la presente disertación se han trazado los siguientes objetivos

1. Realizar una revisión de información en las bases de datos Scielo, Dialnet y Eric relacionada con aportes del cine para la enseñanza y de la ciencia ficción como mediador didáctico para la enseñanza de las ciencias naturales.

2. Categorizar la información obtenida y elaborar un análisis de contenido que articule la información adquirida

3. A partir de los documentos seleccionados al final hallar similitudes y diferencias respecto al uso de la ciencia ficción y del cine en el ámbito educativo

Educar en ciencias es una prioridad educativa que de lograrse con éxito y eficacia constituye un elemento básico en la construcción del pensamiento científico y tecnológico, que permitirá que los miembros de la sociedad se apropien de aspectos básicos que les permitan desenvolverse de manera adecuada ante los retos que se les plantean cotidianamente en un mundo cada vez más globalizado. Esto no implica que el aprendizaje de la ciencia únicamente se vea como algo necesario para aprender a manejar y entender los avances tecnológicos, sino que es un elemento básico para construcción del pensamiento científico y tecnológico.

Respecto a la importancia de la enseñanza de las ciencias naturales Mateu (2005) indica que esta favorece la alfabetización científica de los ciudadanos desde la escolaridad temprana, procurando que comprendan conceptos, practiquen procedimientos $y$ desarrollen actitudes que les permitan participar de una cultura analítica y critica ante la información emergente. 
Edición Extraordinaria. p.p. $182-193$

Memorias del VIII Encuentro Nacional de Experiencias en Enseñanza de la Biología y la Educación Ambiental. III Congreso Nacional de Investigación en Enseñanza de la Biología.

Sin embargo; al observar nuestra práctica se encuentra en los educandos un desinterés generalizado por el aprendizaje de la ciencia; lo cual convoca a plantear los siguientes cuestionamientos; ¿Cuál es la causa de este desinterés y/o el mal funcionamiento de los canales de transmisión de la cultura científica? $O$ es que irealmente los docentes y científicos no sabemos divulgar la ciencia? es preocupante el hecho de que la respuesta al primer interrogante sea qué NO hay desinterés y más bien el segundo interrogante sea un SI; donde docentes y científicos no hemos logrado transmitir la ciencia. (Palacios S, 2006, p. 106).

En la actualidad, dentro de la enseñanza de las ciencias se considera importante hacerlas llegar a todos los alumnos como algo útil, relacionado con la vida real y enseñar una ciencia escolar relevante para el ciudadano (Acevedo, 2004).

Ahora bien no podemos reducir el problema a una simple transmisión de saberes, sino a la manera como logramos los docentes en los estudiantes la construcción del pensamiento científico que les permita desarrollar habilidades y destrezas en las ciencias.

Para intentar paliar en lo posible y/o revertir esta situación se requieren nuevas formas motivadoras a la hora de divulgar, transmitir y enseñar las disciplinas científicas tales como la Física, biología, y otras (Amengual, 2005) uno de estos recursos bien puede ser el cine y la literatura de ciencia ficción, un tema que posee un gran poder de atracción entre los jóvenes y los no tanto (Barceló, 2000; Barceló, 2005)

Aparicio indica que por su carácter lúdico, por su alta pregnancia, por su capacidad de identificación entre espectador y personajes, y por muchos motivos más, el cine es una herramienta idónea para ver y pensar el mundo.

Pérez Vallejo (2010) señala en su artículo titulado "el cine como recurso educativo" las siguientes bondades:

El cine facilita la observación de fenómenos, favorece la percepción y la comprensión, refuerza la atención, produce sensaciones afectivas en los espectadores, sensibiliza ante un determinado problema y ofrece posibilidades de evasión....El cine puede servir como documento de reflexión y debate (...) el cine como comenta MacLuhan en "el aula son muros", complementa conocimientos, integra ideas y lenguajes. El cine puede hacer comprender la vida, inicia a nuevos lenguajes y acerca a los más pequeños conceptos y valores difíciles de apreciar por los medios escolares tradicionales (...) puesto que estamos hablando en términos de educación, cabe destacar que el cine tiene dos facetas didácticas. Una seria la enseñanza del cine como tal, como medio expresivo que tiene un lenguaje propio, y la otra será el aprendizaje que se puede extraer del cine, es decir, el 
Edición Extraordinaria. p.p. $182-193$

Memorias del VIII Encuentro Nacional de Experiencias en Enseñanza de la Biología y la Educación Ambiental. III Congreso Nacional de Investigación en Enseñanza de la Biología.

uso de una película como ilustración o como punto de partida de cualquier centro de interés. Sin que el cine llegue a constituirse en un sustitutivo de la lectura.

Roman Gubern (1996) afirma que el cine "es un instrumento de comunicación de masas que pretende llegar al mayor número de posible de seres humanos". Su objetivo es plural: narra, representa, influye, comunica, informa, enseña, crea y recrea modelo (...). Además de integrar la pluralidad de sentidos a partir de los distintos lenguajes que entran en juego como los sentidos de la vista y el oído, supone también la incorporación de otros lenguajes como la música, los colores, las formas, la fotografía, la luz, etc (Radetich 2011) Lo que le otorga al cine un sentido de transversalidad y de múltiples posibilidades desde diferentes campos de formación.

Luego de haber explorado las bondades que tiene el uso del cine para la enseñanza y como recurso pedagógico, expondremos las fortalezas del aprovechamiento de la ciencia ficción en la escuela. Sierra (2007) indica que es desconcertante el precario uso que se hace de la ciencia ficción para la enseñanza y divulgación de las ciencias, incluida su dimensión axiológica, por lo que propone la puesta en práctica de la ciencia ficción dura, tanto en su versión literaria como en la cinematográfica, para tal enseñanza y divulgación.

Umberto eco (2004)) reseña que las bondades de la ciencia ficción estriban en que trata siempre de imaginar las soluciones posibles de datos actuales, además de desembocar en una crítica positiva.

Asimismo Barcelo (2003) indica; si, como se nos dice tantas veces, el sistema educativo pretende, entre otras cosas, desarrollar nuevas metodologías para contrarrestar el aprendizaje repetitivo y monótono del conocimiento científico, la ciencia ficción puede ser una herramienta importante para lograrlo. Y no solo eso. También puede ayudar para desarrollar actividades interdisciplinarias e integradoras y fomentar la realización de trabajos de síntesis y de proyectos de investigación sugerentes, didácticos $y$, además, francamente divertidos".

De esta forma se integran estos dos componentes por un lado las bondades del cine en la enseñanza y por el otro las ventajas de la ciencia ficción desde el cine, la literatura, los comics o las series de tv y sus múltiples usos en el ámbito educativo; la revisión de las bases de datos nos permitieron hacer la revisión en especial de cinco artículos donde se encuentran fuertemente integrados estos componentes y se expresa los objetivos y resultados de estas investigaciones

Borrás (2006) resalta desde una perspectiva netamente didáctica las posibilidades e incidencias que la ciencia-ficción puede tener en el aula. Indica que el período de la adolescencia encuentra en la ciencia-ficción una forma de explicar sus necesidades y sus ideas previas. Por tanto, para facilitar sus procesos de aprendizaje, al mismo tiempo que 
Edición Extraordinaria. p.p. $182-193$

Memorias del VIII Encuentro Nacional de Experiencias en Enseñanza de la Biología y la Educación Ambiental. III Congreso Nacional de Investigación en Enseñanza de la Biología.

los profesores pueden ver enriquecida su metodología de enseñanza, se muestran diversas fórmulas de acercamiento al alumno por medio de un motivador entorno de aprendizaje.

Carretero G. (2008) desarrolla un trabajo donde centra en la lectura de textos de ciencia ficción las clases de ciencias, el objetivo más importante de esta experiencia ha sido conseguir que los alumnos desarrollen la capacidad de relacionar conceptos que aparecen en la lectura con los conocimientos adquiridos previamente en clase.

En la revista EUREKA en el artículo "El cine y la literatura de ciencia ficción como herramientas didácticas en la enseñanza de la física: una experiencia en el aula"; Palacios (2007) propone como recursos motivador y didáctico tanto la literatura como el cine de Ciencia Ficción, describe la metodología empleada en la asignatura de libre elección ¿Física en la Ciencia Ficción?, incluida en el plan específico de la Facultad de Ciencias de la Universidad de Oviedo, proporcionando asimismo un listado con material de tipo bibliográfico y audiovisual que puede ser utilizado. La experiencia puede ser adaptada fácilmente a la enseñanza de otras disciplinas científicas como pueden ser la química, la biología, etc., tanto en la universidad como en la educación secundaria.

Levin y Almiron (2012) en la revista iberoamericana de educación presentan algunos elementos conceptuales para repensar la utilidad del cine, en particular del cine de ciencia ficción, en la enseñanza de las ciencias, ofrecen una estrategia didáctica, fuertemente anclada en el desarrollo conceptual cuya principal fortaleza es la capacidad de insertar la enseñanza de la ciencia en contextos sociales, materiales y políticos en su metodología; desarrollan una estrategia que llaman "prototipo diegético".

Petit y Solbes (2012) en "La ciencia ficción y la enseñanza de las ciencias"; muestra una primera aproximación a la ciencia en la ciencia ficción desde el punto de vista del alumnado y qué posibilidades ofrece como recurso didáctico. Sondea, por tanto, si los alumnos conocen y han visto cine de ciencia ficción, así como si este factor influye en sus actitudes hacia la ciencia y los científicos y su opinión sobre las consecuencias de los avances científicos en el futuro, plantea si los estudiantes perciben las visiones deformadas de la ciencia y los científicos que transmite el cine de ciencia ficción.

\section{METODOLOGÍA}

El presente trabajo se presenta en el marco de la investigación cualitativa y se realiza un análisis de contenido. Kripppendorff (1980) define el análisis de contenido como "la técnica destinada a formular a partir de ciertos datos, inferencias reproductibles y validas que puedan aplicarse en un contexto". Gomez M. señala que el análisis de contenido se trata de un método que consiste en clasificar $y / 0$ codificar los diversos elementos de un mensaje en categorías con el fin de hacer aparecer de manera 
Edición Extraordinaria. p.p. $182-193$

Memorias del VIII Encuentro Nacional de Experiencias en Enseñanza de la Biología y la Educación Ambiental. III Congreso Nacional de Investigación en Enseñanza de la Biología.

adecuada su sentido y presenta unas características que la distinguen de otras metodologías de investigación sociológica, en que se trata de una técnica que combina intrincadamente, y de ahí su complejidad, la observación y el análisis documental (citado por Gomez M., tomado de Lopez-aranguren 1986).

Gomes M. reseña además a L'Écuyer (1987:50), Landry (1988:329), Mayer y Quellet (1991), quienes precisan las características generales del análisis de contenido en los siguientes términos:

(1) Se trata de una técnica indirecta, porque se tiene contacto con los individuos solo mediante los sesgos de sus producciones, es decir, con los documentos de los cuales se puede extraer información.

(2) Estas producciones pueden tomar diversas formas: escrita, oral, imagen o audiovisual, para dar cuenta de sus comportamientos y de sus fines.

(3) Los documentos pueden haber sido constituidos por una persona, por ejemplo las cartas personales, las novelas, un diario íntimo, o por un grupo de personas, por ejemplo las leyes, los textos publicitarios.

(4) El contenido puede ser no cifrado, es decir, las informaciones que contienen los documentos no se presentan bajo la forma de números sino ante todo de expresiones verbales.

(5) Es posible una deducción cualitativa o cuantitativa. En este sentido, los documentos pueden ser analizados con el objeto de cuantificar o en la perspectiva de un estudio cualitativo de elementos singulares, o los dos a la vez.

En cuanto a la clasificación del análisis de contenido autores como Mayer y Quellet (1991) delimitan seis tipos los cuales se indican a continuación:

1. El análisis de exploración del contenido

2. El análisis de verificación del contenido

3. El análisis de contenido cuantitativo

4. El análisis de contenido directo

5. El análisis de contenido cualitativo

6. El análisis de contenido indirecto

Este trabajo se encuadra en los dos últimos tipos; el análisis de contenido cualitativo y el análisis de contenido indirecto, en el primero ya que este tipo de análisis permite verificar la presencia de temas, de palabras o de conceptos de un contenido como lo es 
Edición Extraordinaria. p.p. $182-193$

Memorias del VIII Encuentro Nacional de Experiencias en Enseñanza de la Biología y la Educación Ambiental. III Congreso Nacional de Investigación en Enseñanza de la Biología.

la CIENCIA FICCION Y LA ENSEÑANZA para nuestro caso; y el segundo ya que el investigador busca extraer el contenido latente detrás del contenido manifiesto, recurrirá a una interpretación del sentido de los elementos, su frecuencia, sus asociaciones, etc. que es finalmente la intencionalidad del presente trabajo.

Para la realización del trabajo se siguieron los siguientes pasos; primero se determinaron los objetivos que se pretendían alcanzar los cuales están señalados previamente en el escrito, luego se seleccionaron las bases de datos Dialnet, Scielo y Eric para consultar la información (artículos) que iban a suministrar la materia prima para la realización del análisis de contenido, la ventana de observación que se establece corresponde a un periodo de tiempo no mayor a 10 años esto debido a que en un análisis previo se encontró que la información disponible respecto al tema analizado es abundante y seria complejo el análisis de esa cantidad de documentos, teniendo claro esto, se procedió a realizar la consulta de las bases de datos, obteniendo lo siguiente:

Primera revisión

\begin{tabular}{|l|l|l|}
\hline Descriptor & Base de datos & Total artículos encontrados \\
\hline \multirow{2}{*}{$\begin{array}{l}\text { Enseñanza de las ciencias y } \\
\text { ciencia ficción }\end{array}$} & Dialnet & 25 \\
\cline { 2 - 3 } & Eric & 112 \\
\cline { 2 - 3 } & Scielo & 0 \\
\hline
\end{tabular}

Segunda revisión

\begin{tabular}{|l|l|l|}
\hline Descriptor & Base de datos & Total artículos encontrados \\
\hline \multirow{2}{*}{$\begin{array}{l}\text { Ciencia ficción } \\
\text { aprendizaje }\end{array}$} & Dialnet & 14 \\
\cline { 2 - 3 } & Eric & 263 \\
\cline { 2 - 3 } & Scielo & 0 \\
\hline
\end{tabular}

Tercera revisión

\begin{tabular}{|l|l|l|}
\hline Descriptor & Base de datos & Total artículos encontrados \\
\hline Ciencia ficción y enseñanza & Dialnet & 26 \\
\cline { 2 - 3 } & Eric & 432 \\
\cline { 2 - 3 } & Scielo & 1 \\
\hline
\end{tabular}

Cuarta revisión

\begin{tabular}{|l|l|l|}
\hline Descriptor & Base de datos & Total artículos encontrados \\
\hline El cine de ciencia ficción & Dialnet & 11 \\
\cline { 2 - 3 } $\begin{array}{l}\text { para la enseñanza de las } \\
\text { ciencias }\end{array}$ & Eric & 0 \\
\cline { 2 - 3 } & Scielo & 15292 \\
\hline
\end{tabular}

Luego de hacer la revisión en las bases de datos cambiando los descriptores con el ánimo de reducir la cantidad de artículos encontrados, guardando relación con el sentido de la propuesta de trabajo de grado se seleccionaron en primer lugar artículos que 
Edición Extraordinaria. p.p. $182-193$

Memorias del VIII Encuentro Nacional de Experiencias en Enseñanza de la Biología y la Educación Ambiental. III Congreso Nacional de Investigación en Enseñanza de la Biología.

correspondían a "El cine de ciencia ficción para la enseñanza de las ciencias"; sin embargo par la base de datos Eric la cual siempre arrojaba una cantidad amplia de documentos se consideró el descriptor "Enseñanza de las ciencias y ciencia ficción", lo cual no afecto de ninguna manera el análisis documental dado que dentro de los 15292 artículos relacionados con el cuarto descriptor están contenidos los 112 del primer descriptor, pero al ser menor el número de artículos permite un mejor tratamiento de la información. A continuación se relacionan varios de los artículos encontrados (no corresponden a la totalidad para la base de datos Eric por ser amplia la cantidad de artículos)

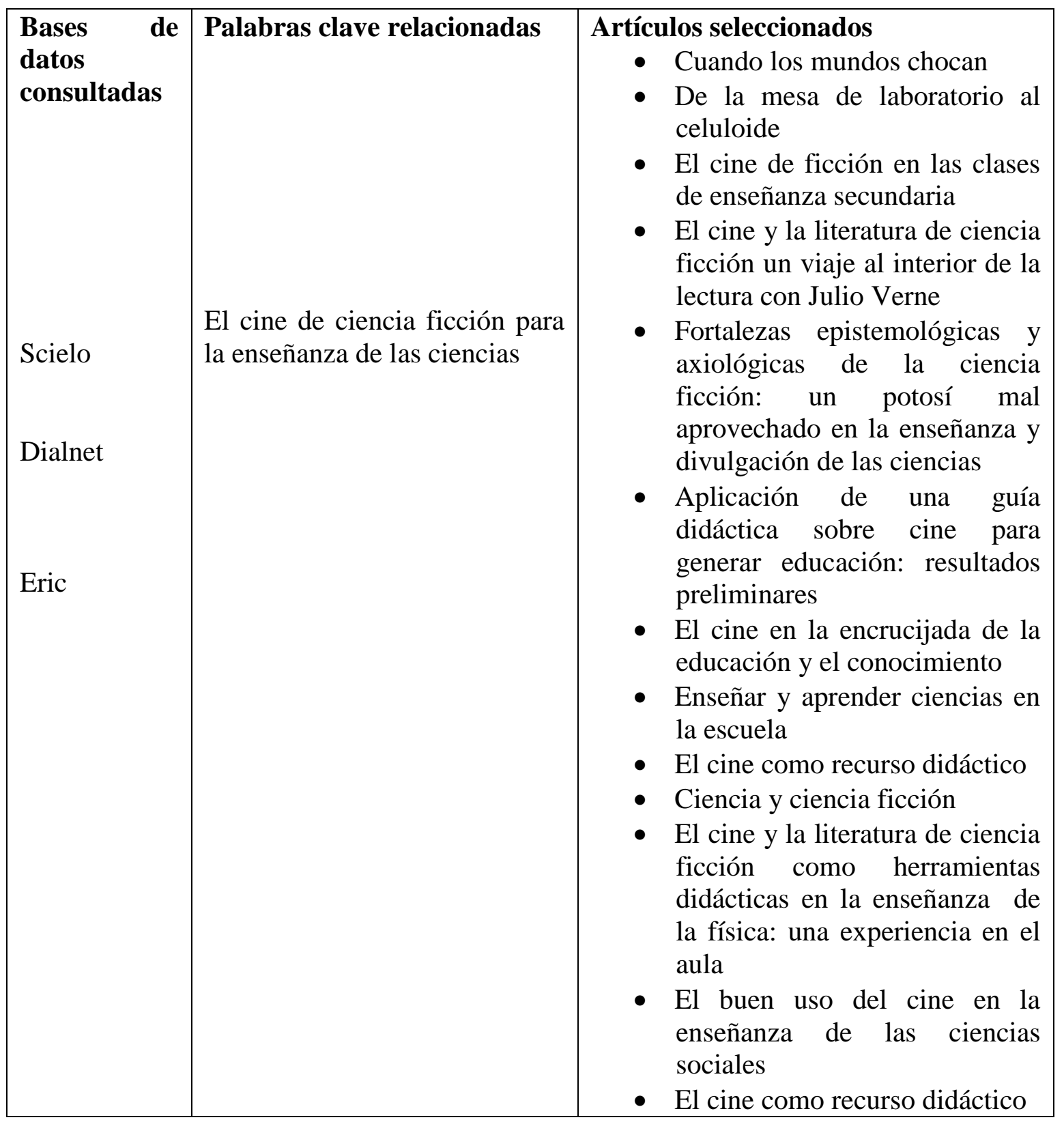


Edición Extraordinaria. p.p. $182-193$

Memorias del VIII Encuentro Nacional de Experiencias en Enseñanza de la Biología y la Educación Ambiental. III Congreso Nacional de Investigación en Enseñanza de la Biología.

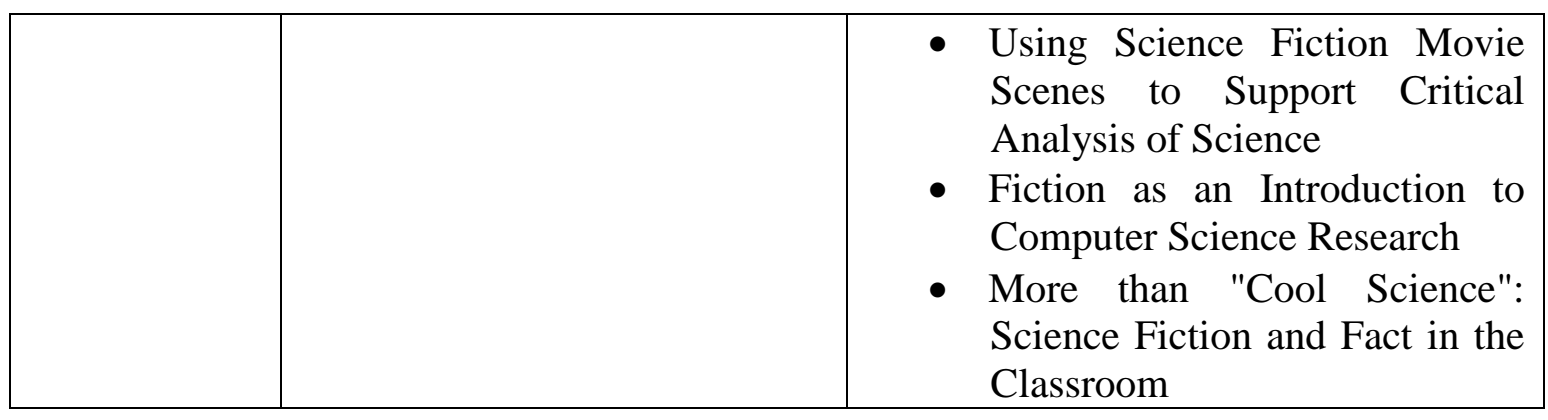

Luego se llevó a cabo la categorización de la información seleccionada teniendo presente que las categorías que se establecieron guardan una profunda relación con la naturaleza del proyecto de grado. Para dearrollar este proceso se analizaron los distintos documentos y se establecieron similitudes respecto a dos condiciones contenidas y desarrolladas en los documentos seleccionados y estas son: EL CINE COMO INSTRUMENTO PARA LA EDUCACIÓN Y LA CIENCIA FICCIÓN COMO MEDIADOR DIDÁCTICO. Fueron seleccionados cinco documentos, los cuales desarrollaban las categorías seleccionadas

Borrás (2006). Cuando los mundos chocan. Revista EUREKA sobre enseñanza y divulgación de las ciencias.

Carretero, G. M.B., (2008). Un viaje al interior de la lectura con Julio Verne. Revista EUREKA sobre enseñanza y divulgación de las ciencias.

Levin, L., Arango C.B., y Almirón, M.E., (2012). De la mesa de laboratorio al celuloide: el cine en la enseñanza de las ciencias. Revista iberoamericana de educación.

Palacios S.L., (2007). El cine y la literatura de ciencia ficción como herramientas didácticas en la enseñanza de la física: una experiencia en el aula. Revista EUREKA sobre enseñanza y divulgación de las ciencias.

Petit M.F. y Solbes M.J. (2012). La ciencia ficción y la enseñanza de las ciencias. Enseñanza de las ciencias Revista de investigación y experiencias didácticas. 
Edición Extraordinaria. p.p. $182-193$

Memorias del VIII Encuentro Nacional de Experiencias en Enseñanza de la Biología y la Educación Ambiental. III Congreso Nacional de Investigación en Enseñanza de la Biología.

\section{CONCLUSIONES}

Luego de haber analizado los documentos seleccionados se realizaron los siguientes hallazgos:

$\checkmark$ La ciencia ficción es un recurso inagotable de posibilidades y aunque diversos autores lo trabajan cada uno de ellos tiene una visión distinta de un mismo recurso, mostrando las bondades y cualidades de este género; así Petit y Solves utilizan la CF para analizar las aptitudes para la ciencia, las visiones deformadas de la ciencia y de los científicos; mientras que Levin y Almirón la utilizan como estrategia didáctica que posibilita la construcción conceptual, Borrás resalta desde una perspectiva netamente didáctica, las posibilidades e incidencias que la ciencia-ficción puede tener en el aula, Palacios propone como recursos motivador y didáctico tanto la literatura como el cine de Ciencia Ficción y Carretero Gómez se centra en la lectura de textos de ciencia consiguiendo que los alumnos desarrollen la capacidad de relacionar conceptos que aparecen en la lectura con los conocimientos adquiridos previamente en clase.

$\checkmark$ A pesar de que la ciencia ficción ofrece múltiples posibilidades para su desarrollo y puesta en marcha desde diferentes ámbitos como lo son el cine, la literatura, los comics y las series de televisión, la mayoría de artículos y documentos encontrados utilizaron el cine como instrumento para el desarrollo de sus trabajos según plantean los mismos este facilita la observación, favorece la comprensión y la percepción, mejora la atención y sensibiliza ante un determinado problema.

$\checkmark$ La ciencia ficción causa una especial fascinación entre los jóvenes y los adultos por tal razón favorece el aprendizaje convirtiéndose en un eficaz mediador y motivador didáctico, además el poderse trabajar desde el ámbito de la literatura favorece las competencias comunicativas y lectoras.

$\checkmark$ A nivel de Colombia son escasos los trabajos, escritos o artículos relacionados con este recurso y aquellos que se encontraron corresponden a la enseñanza de la física, además no existen políticas por parte del MEN o de la SED que favorezcan o potencien el uso de la CF o del cine en las escuelas o para la enseñanza; situación que si sucede en otros países como España en donde incluso existe un curso que permite que cualquier docente de cualquier campo disciplinar se capacite para incluir el cine como recurso didáctico. 
Bio - grafía. Escritos sobre la Biología y su Enseñanza. ISSN 2027-1034

Edición Extraordinaria. p.p. $182-193$

Memorias del VIII Encuentro Nacional de Experiencias en Enseñanza de la Biología y la

Educación Ambiental. III Congreso Nacional de Investigación en Enseñanza de la Biología.

\section{BIBLIOGRAFIA}

Aguilar F. (2011). Propuesta didáctica para la enseñanza y aprendizaje de conceptos de densidad y presión abordados en la educación básica secundaria. Trabajo de tesis para optar al título de Magister. Universidad Nacional de Colombia.

Barceló, M. (2003). Ciencia y ciencia ficción. Quark: ciencia, medicina, comunicación y cultura, ISSN 1135-8521 ( $N^{0}$ 28-29) recuperado de http://quark.prbb.org/2829/028097.htm

Carmona, A. (2009). La investigación acción en la enseñanza de la física: un escenario idóneo para la formación y desarrollo profesional del profesorado. Recuperado de http://www.lajpe.org/may09/29 Antonio Garcia.pdf

Carretero G. M. (2008). Un viaje al interior de la lectura con Julio Verne. Revista EUREKA sobre enseñanza y divulgación de las ciencias. SSN-e 1697-011X, Vol. 5, No. 3 , 2008, págs. 302-313

García Borras, F.J. (2006). Cuando los mundos chocan. Revista EUREKA sobre enseñanza y divulgación de las ciencias. ISSN-e 1697-011X, Vol. 3, Nº. 2, págs. 268-286

García, R. M. \& Orozco R.L. (2008). Orientando un cambio de actitud hacia las ciencias naturales y su enseñanza en profesores de educación primaria. Revista electrónica de enseñanza de las ciencias. Vol 7. № 3, pags. $539-568$.

Levin, L., Arango, C \& Almiron M. (2012). De la mesa de laboratorio al celuloide: el cine en la enseñanza de las ciencias. Revista iberoamericana de educación. ISSN-e 10226508, N 58, 2012, págs. 99-117

Marquez, B. C. (2005). Aprender ciencias a través del lenguaje. Recuperado de http://gent.uab.cat/conxitamarquez/sites/gent.uab.cat.conxitamarquez/files/Aprender $\% 20$ ciencias $\% 20 a \% 20$ traves $\% 20$ del\%20lenguaje_0.pdf

Mora M., Aguilar F. (2011). Propuesta didáctica para la enseñanza y aprendizaje de conceptos físicos básicos a partir del uso del video de ciencia ficción y prácticas de aula demostrativas. Revista científica ISNN -e 2344 - 8350, No 13 . Recuperado de http://revistas.udistrital.edu.co/ojs/index.php/revcie/article/view/1313

Palacios, S. (2007). El cine y la literatura de ciencia ficción como herramientas didácticas en la enseñanza de la física: una experiencia en el aula. Cuando los mundos chocan. Revista EUREKA sobre enseñanza y divulgación de las ciencias. ISSN-e 1697011X,Vol. 4, Nº. 1, 2007, págs. 106-122 
Bio - grafía. Escritos sobre la Biología y su Enseñanza. ISSN 2027-1034

Edición Extraordinaria. p.p. $182-193$

Memorias del VIII Encuentro Nacional de Experiencias en Enseñanza de la Biología y la

Educación Ambiental. III Congreso Nacional de Investigación en Enseñanza de la Biología.

Petit, M., (2012). La ciencia ficción y la enseñanza de las ciencias. Enseñanza de las ciencias: revista de investigación y experiencias didácticas. ISSN0212-4521, Vol. 30, $N^{\circ} 2,2012$, págs. 55-72

Rojas, G. A. (2009) la investigación - acción en el aula. Reflexiones de profesionales de la educación. Recuperado de http://www.oei.es/metas2021/reflexiones2/10.pdf

Sierra C. E. (2007). Fortalezas epistemológicas y axiológicas de la ciencia ficción: un potosí pedagógico mal aprovechado en la enseñanza de las ciencias. Revista EUREKA sobre enseñanza y divulgación de las ciencias. ISSN-e 1697-011X,Vol. 4, №. 1, 2007, págs. 87-105 